\title{
Postoperative cure in Iranian patients with gastric cancer: estimating the crude conditional probability in a relative survival setting in the presence of competing risks
}

\author{
Fatemeh Paknazar", Mahmood Mahmoudi*, Kazem Mohammad, Hojjat Zeraati, \\ Mohammad Ali Mansournia, Mehdi Yaseri
}

Abstract

Background: Following treatment, cancer patients may be clinically cured. However, they may die for reasons other than cancer, called competing risks.

Objective: To estimate postoperative cure while considering the competing risks in Iranian patients with gastric cancer. Method: Data were obtained from the Cancer Institute of Imam Hospital in Tehran. The analysis was conducted within the framework of relative survival by fitting the data to a flexible parametric cure model, taking into account the competing risks using general population data by adjusting for age, sex, and year of diagnosis.

Results: Of the 326 patients (224 male and 102 female) whose data were included, 235 deaths (72.1\%) occurred during the follow-up period. The probability of conditional cure in terms of crude ratios of dying from causes other than gastric cancer in the surviving patients increased with the passage of time, and the slope of excess mortality approached almost 0 after 7 years. The estimated cure ratios showed a variation from $69 \%$ for 50 -year-old men with diagnosis at early stages (I and II) to 3\% for 80-year-old women with diagnosis at stage IV.

Conclusion: The ratio of patients in Iran who were estimated to die from cancer reduced significantly with the passage of time following the diagnosis, and the statistical cure point was estimated to be 7 years after diagnosis. However, aging was shown to be inversely associated. Although the same trend was observed in both sexes, we showed that men were statistically more likely to reach the cure point.

Keywords: competing risk, cure, gastric cancer, Iran, relative survival

Gastric cancer, a major health problem, is the fourth most common cancer in the world, and more than $70 \%$ of cases of gastric cancer occur in developing countries, especially in Asian countries $[1,2]$. In Asia, following breast and lung cancers, gastric cancer is ranked the third in terms of the incidence [3]. Despite the decline in the incidence of the disease in recent decades, it is still considered as the second leading cause of mortality from cancer in Asia (ranked after lung cancer) [2-4].

*Correspondence to: Mahmood Mahmoudi, Department of Epidemiology and Biostatistics, School of Public Health, Tehran University of Medical Sciences, Tehran, Iran, e-mail: mahmoodim@tums.ac.ir

Department of Epidemiology and Biostatistics, School of Public Health, Tehran University of Medical Sciences, Tehran, Iran

"Nursing Care Research Center, Semnan University of Medical Sciences, Semnan, Iran

¿ Open Access. ๑ 2017 Fatemeh Paknazar et al., published by Sciendo. (c) BY-NC-ND This work is licensed under the Creative Commons Attribution NonCommercial-NoDerivatives 4.0 License. 
There are individuals of various ethnicities living in the Middle East region, including Arabs, Jews, Persians, Kurds, Turks, and Turkmen. Because of these various ethnicities, the prevalence of cancer in the countries of this region ranges from extremely high in Iran (26.1 per 100,000 people) to low in Egypt (4.8 per 100,000 people) $[3,5,6]$. The incidence of gastric cancer in Iran is 7 times higher than that in the neighboring country, Iraq [7]. Iran, with 14.1 deaths per 100,000 people each year, has the highest mortality from cancer among these countries [3, 5, 8]. Based on the SEER database, the relative survival of patients with gastric cancer was $27 \%$ between 2002 and 2008 [9]. Based on the results of a study conducted in Tehran of 760 patients with gastric cancer (from 1993 to 2006 ), the 1 - and 5 -year survival of the studied patients was $52 \%$ and $24 \%$, respectively [10]. In the mentioned study, 326 (43\%) patients were diagnosed at stages 1 and 2, and the rest were diagnosed at stages 3 and 4 [10]. In another study conducted of 128 patients with gastric cancer in the Hamadan province in Iran (from 2007 to 2013), 1-, 2-, and 3-year survival rates were $51 \%, 13 \%$, and $5 \%$, respectively [11]. In the mentioned study, only 40 (31\%) patients were diagnosed at stages 1 and 2 [11]. In both the mentioned studies, age and disease stage were reported as the 2 factors affecting the survival of patients $[10,11]$. Histopathologically, the most common form of the disease was adenocarcinoma $[6,9,12]$.

A considerable proportion of studies on cancer are dedicated to analyzing the survival of patients with cancer. In survival analysis, the survival time from the onset of disease to death can be considered as the most useful indicator to evaluate the status of diagnosis and treatment of the disease. In general, there are two types of survival measures: crude survival measure and net survival measure. Even though the crude survival measure is estimated through completely real-world probabilities with any cause of death, the net survival measure is estimated through hypothetical global probabilities or unrealistic scenarios [13]. It is important to recognize that net survival is interpreted for a hypothetical world where competing risks are assumed to be eliminated. The cancer-specific survival in the presence of competing risks is often higher than the cancer-specific survival in the absence of competing risks. If we assume that a type of cancer is the only cause of death in a community, then the net survival of the patients with the cancer after the diagnosis will be equal to the percentage of patients who are still alive after a certain period (for example, 5 years). There are two settings for estimating the net and crude survival in patients with cancer, cause-specific and relative survival. One of the most practical is the relative survival setting $[13,14]$. Relative survival, an estimate of pure survival, is the ratio of survival observed in patients to the expected survival in a community.
For many cancers, after adopting effective treatment measures and generally after few years of diagnosis, the relative survival approaches 1 , which statistically means that the risk of death in the patients is not greater than the risk in the nonaffected population, or in other words, the patients are cured. This point of time is known as the "statistical cure point," which can be estimated using various statistical models called "cure models" $[15,16]$. However, because of the limitations in the flexibility of many models, especially for older age groups, their use may be associated with defects and errors. For this reason, we should either remove the data on older age groups or be more cautious when interpreting the data on these age groups. Flexible parametric cure models are superior to other models because they can be used to include and analyze older age group with the least concern [17].

Following definitive treatment, patients with local types of gastric cancer may probably be cured. Currently, the only option for a definitive treatment is surgery $[6,10,11]$. However, patients with cancer may die at any time for reasons other than cancer, which are called competing risks [17]. We sought to answer the following question: what is the probability of ultimately dying from causes other than gastric cancer for an Iranian patient (male/female) who has been diagnosed with gastric cancer at a particular age in a particular year and undergone surgery in Iran immediately after diagnosis (and, of course, received subsequent treatments) and survived for a given period? The answer to this question is found by estimating the ratio of cure by adjusting for the competing risks [17]. The present study aimed to estimate the crude cure probability, while considering the competing risks in Iranian patients with gastric cancer who had undergone gastrectomy.

\section{Methods}

This was a retrospective single-center study performed using data obtained from patients diagnosed with gastric cancer who were referred to the Cancer Institute of Imam Hospital in Tehran and underwent surgery during the time from 1993 to 2000 and were followed up until 2004. The study's inclusion criteria were patients with a pathological diagnosis of gastric cancer who were surgical candidates for total or partial gastrectomy as the first line of action as prescribed by the treatment team and the physician. The exclusion criteria were patients who were not eligible for the surgery or who died during the time of the first hospitalization and those without reliable data regarding their condition.

Flexible parametric cure models were fitted on the log cumulative excess hazard scale using restricted cubic splines to model the baseline excess hazard. The model can predict 
relative (or excess mortality) and cure by adjusting for age, sex, and year of diagnosis taking into account the competing risks $[17,18]$.

Cure was estimated by restricting the cumulative baseline excess hazard function to have zero slope after the last knot. The statistical cure point was defined via the placement of the last knot. Considering the cure time, we determined the determined the trend of mortality by age, sex, and year of diagnosis based on the crude probabilities in the patients, 1, 3, and 5 years after the diagnosis. Then we determined the point and distance estimates for the ratio of cure and ratio of deaths from other causes in the patients based on the statistical end point applying the method used and introduced by Eloranta et al. [17], the analysis was conducted within the framework of relative survival by fitting the flexible parametric cure model, taking into account the competing risks by adjusting for age, sex, and year of diagnosis [18].

In the present study, the outcome was all-cause mortality (regardless of its cause, registered in the patient's profile or death certificate). The relative survival of the patients was estimated by comparing the survival with the life expectancy of a reference population of the same sex and age (based on the year of birth). To obtain data for the life expectancy of the reference population, we used national-level data.

Taking into consideration the concept of crude survival [17], the crude probability of death in patients with cancer during the follow-up was defined in 4 ways: the crude probability of death from cancer $\left(P_{c d c}\right)$, the crude probability of death because of causes other than the diagnosed cancer $\left(P_{c d o}\right)$, the probability of being destined to die ultimately from cancer $\left(P_{b d c}\right)$, and the probability of being destined to die ultimately from causes other than the diagnosed cancer $\left(P_{b d o}\right)$. The crude probability of death from cancer in time $t$ was obtained using the following equation (Eq. 1) [18]:

$$
P_{c d c}(t)=\int_{0}^{t} s^{*}(u) R(u) \lambda(u) d(u)
$$

where $R(u)$ is relative survival, $\lambda(u)$ excess mortality, and $S^{*}(u)$ expected survival in the general population $[19,20]$. In addition, $P_{c d o}(\mathrm{t})$ was calculated by replacing with or expected mortality rate in the general population. Considering that the statistical cure of cancer would occur over time $t$, it would be acceptable to assume that people who survive up to the given time will not die from cancer. In other words, the probability of ultimate death from the diagnosed cancer in patients with cancer will be equal to the probability of death from cancer until $a$, the time (Eq. 2) [17].

$$
P_{b d c}=P_{c d o}(a)
$$

In patients who survived up to the time $t$, the probability of death from the cancer $\left(P_{\text {alive.bdc }}\right)$ and the probability of death because of causes other than the diagnosed cancer $\left(P_{\text {alive.bdo }}\right)$ can be calculated using Equations 3 and 4:

$$
\begin{gathered}
P_{\text {alive. } b d c}(t)=P_{b d c}-P_{c d c}(t) \\
P_{\text {alive. } b d o}(t)=1-P_{c d c}(t)-P_{b d c}
\end{gathered}
$$

To calculate the crude conditional probability of death from other causes in people who survived up to the time $t$, we used Equation 5 [13].

$$
P_{c d o \mid a l i v e}(t)=1-\frac{P_{b d c}-P_{c d c}(t)}{1-P_{c d c}(t)-P_{c d o}}
$$

It is worth noting that $P_{c \text { dolalive }}(t)$ is a concept that is similar to the conditional probability of cure in patients who survived up to the time $t$. Stata 14 was used for statistical analyses.

\section{Results}

Of the 326 patients (224 male and 102 female) whose data were included, $244(74.8 \%)$ patients were at late stages of cancer (stages III and IV) and $82(25.1 \%)$ patients were at early stages of cancer (stages I and II). During follow-up, 235 (72\%) deaths occurred (death from all causes). Most of the patients were diagnosed at later stages, especially in stage IV. The highest mortality during the follow-up of the patients was observed in the same group (150 deaths, approximately $78.5 \%$ of all patients with diagnosis at stage IV). The other characteristics of the patients are presented in Table $\mathbf{1}$.

Taking into account the competing risks in both sexes, the probability of cure in the surviving patients increased with the passage of time from diagnosis. Table 2 presents the conditional cure ratio (predicted probability of death from other causes), which was estimated using a flexible parametric cure model in the presence of competing risks for patients by age (i.e., 50, 60, 70 , and 80 years) and disease stage at diagnosis. The estimated cure ratios showed a variation from $69 \%$ for 50 -year-old men with a diagnosis at early stages (I and II) to $3 \%$.

Figure 1 shows the vital status and probability of death from causes other than cancer 1, 3, and 5 years after the diagnosis in men with gastric cancer (as an example). As shown in Figure 1, the ratio of the patients estimated to die because of cancer is considerably reduced over the passage of time after diagnosis. By contrast, aging was associated with a reduction in the ratio of death from causes other than cancer. We note 
Table 1. Characteristics of the patients with gastric cancer (1993-2000)

\begin{tabular}{lrrr}
\hline Characteristics & No. (\%) & $\begin{array}{r}\text { No. of deaths } \\
\text { (\%) }\end{array}$ & $\begin{array}{r}\text { Percentage } \\
\text { of deaths }\end{array}$ \\
\hline Total & 326 & 235 & 72.1 \\
Stage at diagnosis & & & \\
$\quad$ I & $22(6.7)$ & $13(5.5)$ & 59.1 \\
II & $60(18.4)$ & $35(14.9)$ & 58.3 \\
III & $53(16.3)$ & $37(15.8)$ & 69.8 \\
IV & $191(58.6)$ & $150(63.8)$ & 78.5 \\
Age at diagnosis (years) & & & \\
$\quad<61$ & $87(26.7)$ & $52(22.1)$ & 59.7 \\
$\quad 61-70$ & $122(37.4)$ & $83(35.3)$ & 68.0 \\
$\quad>70$ & $117(35.9)$ & $100(42.6)$ & 85.5 \\
Year of diagnosis & & & \\
$\quad$ Before 1996 & $76(23.3)$ & $55(23.4)$ & 72.4 \\
$\quad 1996-1997$ & $109(33.4)$ & $81(34.5)$ & 74.3 \\
$\quad$ After 1997 & $141(43.3)$ & $99(42.1)$ & 70.2 \\
Sex & & & \\
$\quad$ Male & $224(68.7)$ & $155(65.9)$ & 69.2 \\
$\quad$ Female & $102(31.3)$ & $80(34.1)$ & 78.4 \\
\hline
\end{tabular}

${ }^{\dagger}$ Deaths from all causes
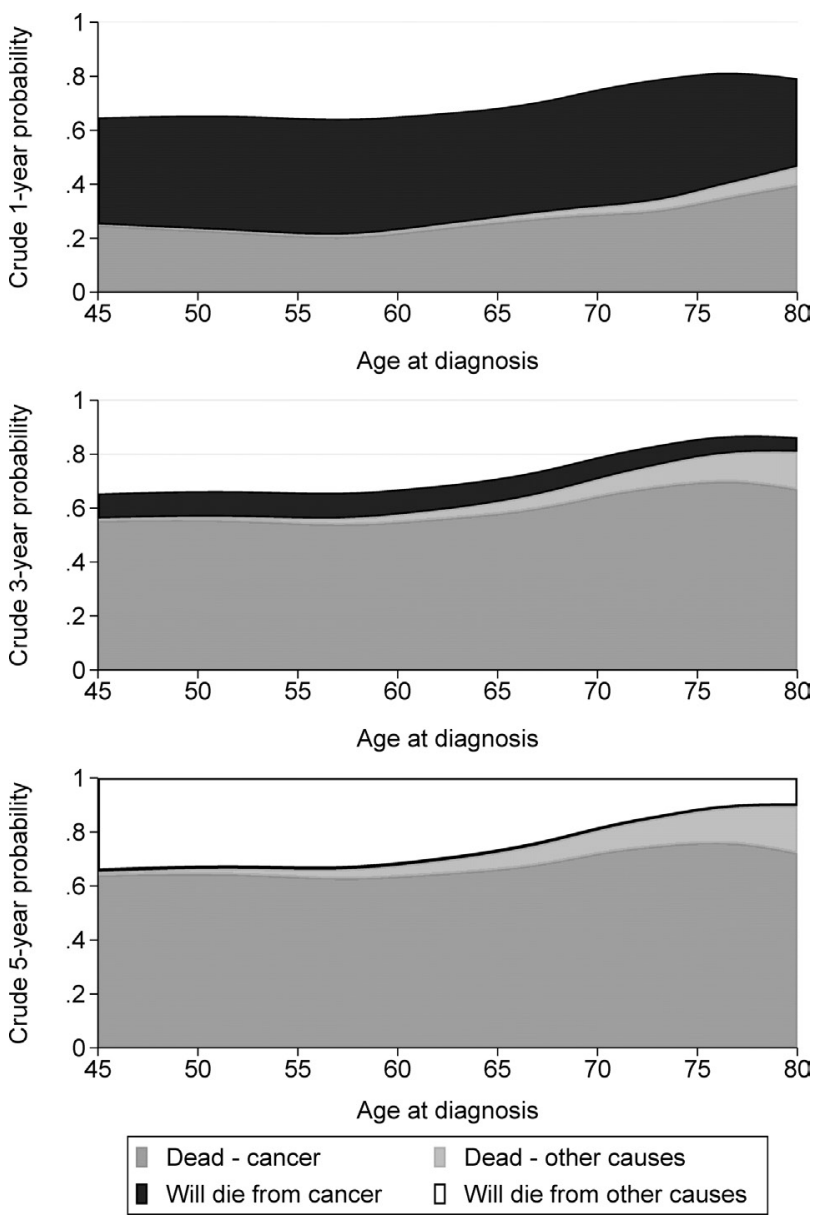

Table 2. Statistical crude ratios of patients who died from causes other than gastric cancer estimated using the flexible parametric cure model in the presence of competing risks by stage and age at diagnosis and sex

\begin{tabular}{lrrr}
\hline \multirow{2}{*}{\begin{tabular}{l} 
Stage at $\begin{array}{c}\text { Age at diagnosis } \\
\text { diagnosis }\end{array}$ \\
\cline { 3 - 4 } (years)
\end{tabular}} & Men & Women \\
\hline I and II & 50 & $0.69(0.46,0.84)$ & $0.63(0.28,0.80)$ \\
& 60 & $0.48(0.31,0.63)$ & $0.45(0.24,0.64)$ \\
& 70 & $0.40(0.18,0.61)$ & $0.25(0.08,0.45)$ \\
& 80 & $0.08(0.02,0.26)$ & $0.05(0.01,0.27)$ \\
III & 50 & $0.49(0.25,0.69)$ & $0.20(0.01,0.54)$ \\
& 60 & $0.45(0.24,0.64)$ & $0.17(0.08,0.29)$ \\
& 70 & $0.28(0.14,0.43)$ & $0.11(0.04,0.25)$ \\
& 80 & $0.07(0.01,0.17)$ & $0.06(0.01,0.25)$ \\
IV & 50 & $0.32(0.19,0.45)$ & $0.27(0.13,0.42)$ \\
& 60 & $0.22(0.14,0.31)$ & $0.12(0.03,0.28)$ \\
& 70 & $0.14(0.08,0.22)$ & $0.10(0.02,0.19)$ \\
& 80 & $0.04(0.01,0.14)$ & $0.03(0.01,0.16)$ \\
\hline
\end{tabular}

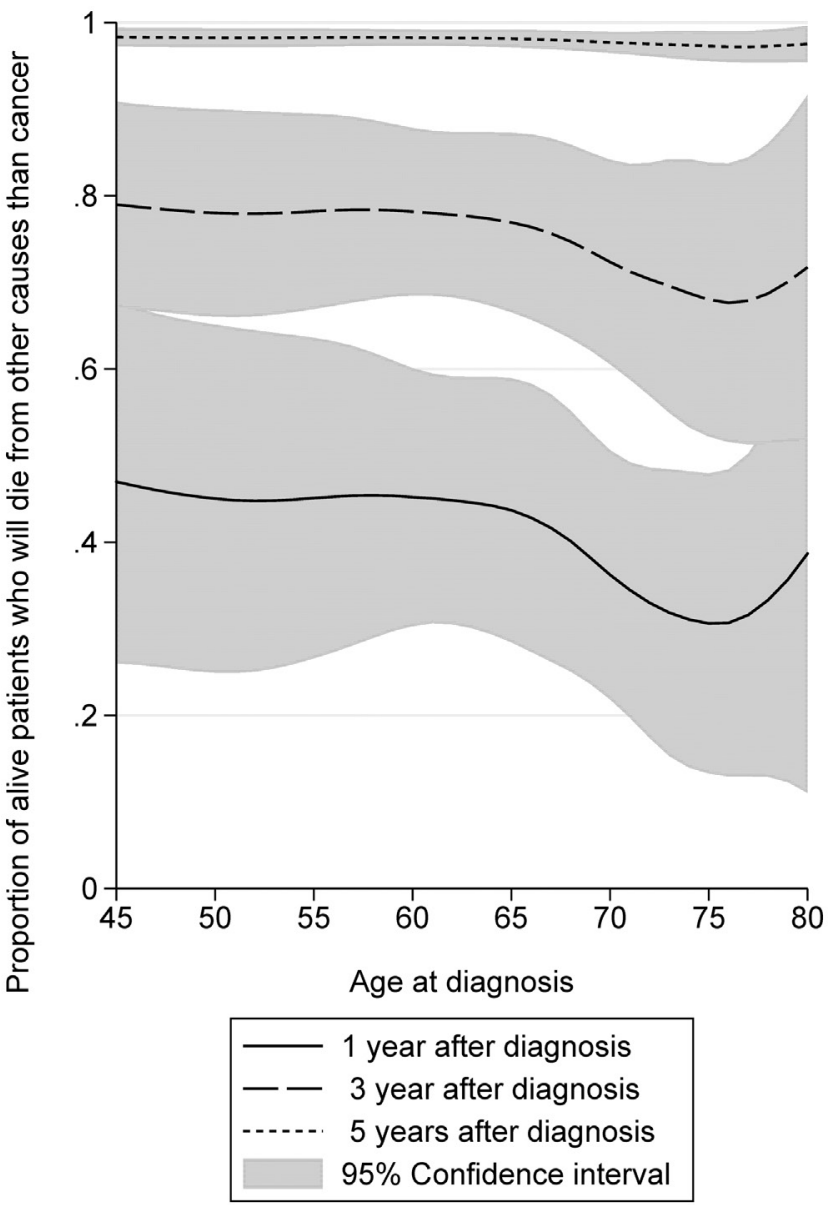

Figure 1. Graph of predicted 1-, 3-, and 5-year vital status (left) and crude ratios of men who will die from causes other than gastric cancer (right) by age at diagnosis (years) 
that because the slope of excess mortality almost approached 0 after 7 years, the statistical cure point in the studied patients was estimated to be 7 years (Figure 2).

As an example, Figure 2 shows the survival status of a 75-year-old Iranian man in the years after diagnosis by the net, crude, and conditional probability (the patient had undergone surgical operation after being diagnosed with gastric cancer in 1999). As shown in Figure 2, with the passage of time that he survives, the conditional probability of death from causes other than gastric cancer approaches 1 . The slope of the graph becomes almost horizontal in the years leading up to the seventh year. This trend indicates that if the patient survives in that time after diagnosis, he/she will approach a cure condition [17].

\section{Discussion}

In the present study, the results of the analysis showed that the ratio of patients who were estimated to die from cancer significantly reduced with the passage of time following the
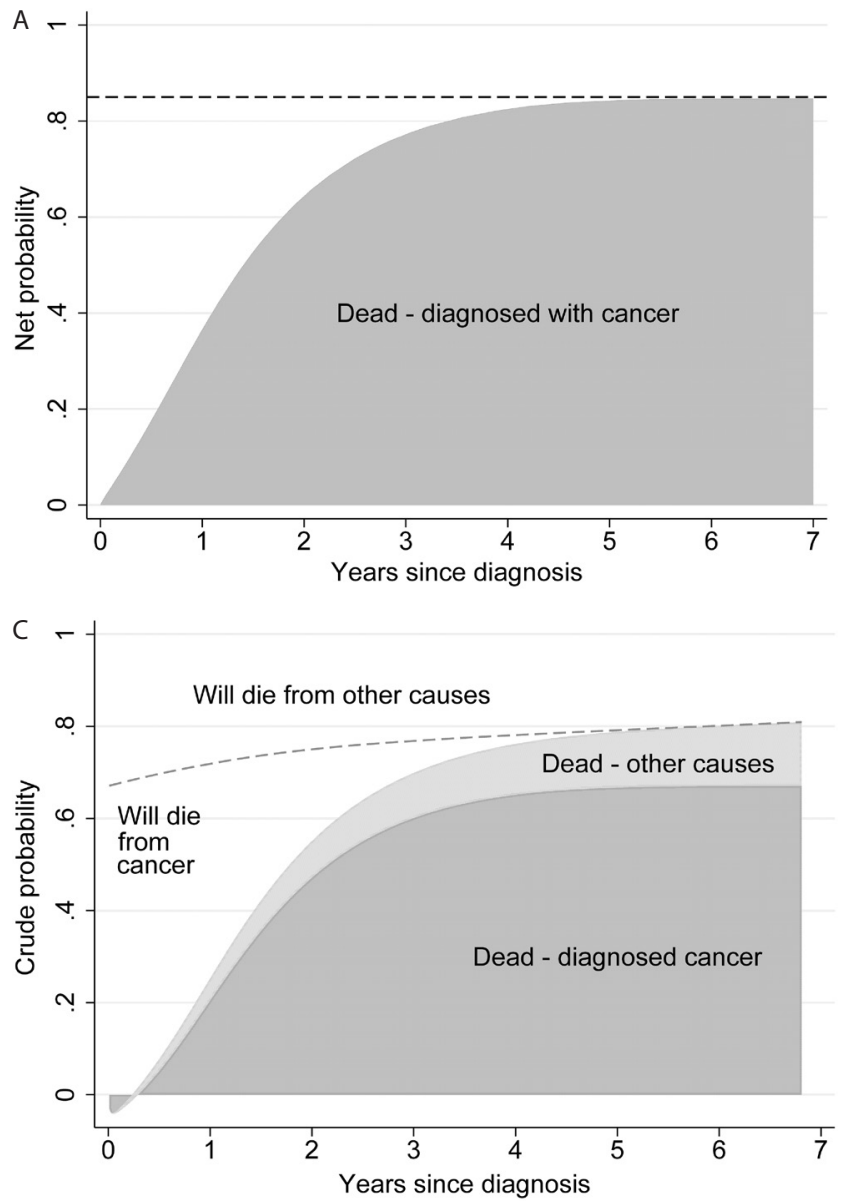

diagnosis. By contrast, aging was associated with a reduction in the ratio of deaths from causes other than gastric cancer. Indeed, when the diagnosis of disease occurs at an older age, there will be a reduction in the ratio of conditional cure (or death from causes other than cancer). For instance, in men with a diagnosis at stage III, the ratio varies from $49 \%$ for 50 -yearold patients to $7 \%$ for 80 -year-old patients. Although the same trend is observed in women, overall, it seems that men are more likely to survive than women. For example, the ratio of women diagnosed at stage III varies from $20 \%$ in 50 -year-old patients to $6 \%$ in 80 -year-old patients. This finding is also true for patients diagnosed at the other stages.

To date, few studies have used our approach to research the survival of patients with cancer. To our knowledge, this method was first used in Sweden to study 3 cancers including colorectal cancer, melanoma, and leukemia [17]. On comparing the results of our study with that of the mentioned study, our findings were largely similar to their findings for colorectal cancer; by contrast, they were different from the findings for melanoma. The observed difference is largely attributable
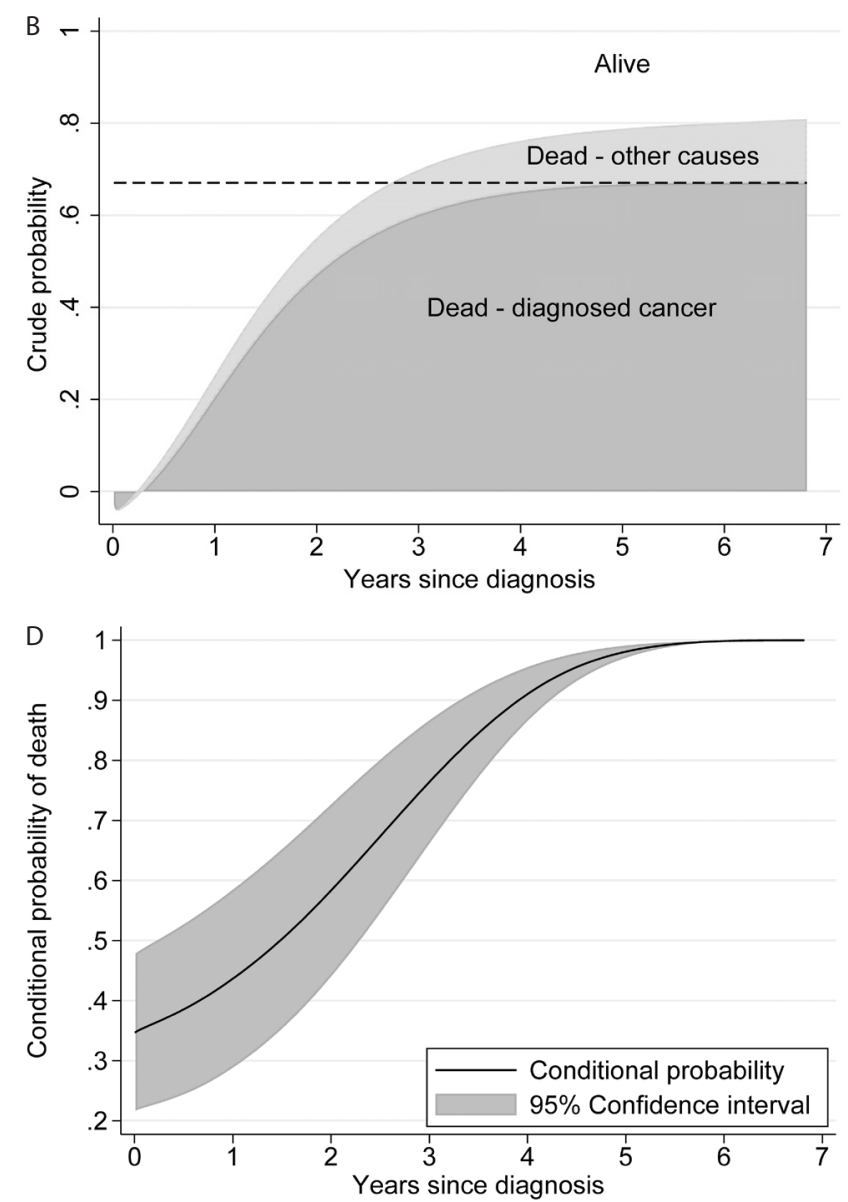

Figure 2. A. Net probability. B. Crude probability. C. Crude probability further partitioned by predicted prognosis. D. Conditional probabilities of death from competing causes 
to the differences in the recurrence of the two diseases after their treatment.

The clinical application of the present study depends on its statistical method. It is usually difficult to determine accurately and record the true cause of death for each disease, and there are always some degrees of error, even in the best and most advanced registration systems. This is also true for cancer. Conversely, when the main cause of death in a cancer patient is apparently something other than the cancer, for example, a traffic accident or myocardial infarct, it cannot be said that the cancer did not play any role in the death of the patient. An important advantage of using the relative survival method as applied through measuring net survival is that the analysis is performed collectively, not individually, based on excess mortality in patients of interest compared with the mortality in the general population of same sex and year of birth. Accordingly, the deaths are not attributable to cancer in a group whose mortality rate is equal to the mortality rate in the reference population; however, the deaths can be partially attributed to cancer when the mortality rate in patients is higher than the mortality rate in the reference population.

Although in this present study the specific cause of death (including death from cancer and noncancer diseases) was not determined, the relative survival of patients was estimated and reported by making a comparison with the life expectancy of Iranian population of the same sex and year of birth. This type of analysis is valuable for clinicians or health practitioners because it helps to estimate the probability of death attributable to cancer and the survival rate at the time of diagnosis (disease stage) without the need to know the precise cause of death and without considering the competing risks that occur with aging after the main treatment and using complementary therapies, and controlling the factors involved in the spread of the disease.

Taking into consideration the limitations of generalizability, the results obtained in this study should be interpreted with caution. The main limitations of this study were the small sample size and the relatively short duration of follow-up. We recommended using this method to conduct survival analysis using large and long-term population data.

\section{Conclusion}

The ratio of patients in Iran who were estimated to die from cancer reduced significantly with the passage of time following the diagnosis, and the statistical cure point was estimated to be 7 years after diagnosis. However, aging was shown to be inversely associated. Although the same trend was observed in both sexes, we showed that men were statistically more likely to reach the cure point.
Author contributions. FP, MM, KM, and MY substantially designed and conceived the study. MM, HZ, and MY acquired the data, and FP, MM, KM, and HZ substantially contributed to their analysis and interpretation. All authors drafted and critically revised the manuscript, approved the final version submitted for publication, and take responsibility for the statements made in the published article.

Acknowledgments. We did not receive any specific grant for this research from any funding agency in the public, commercial, or not-for-profit sectors.

Conflict of interest statement. The authors have each completed and submitted an International Committee for Medical Journal Editors Uniform Disclosure Form for Potential Conflicts of Interest. None of the authors disclose any conflict of interest.

\section{References}

[1] Yang L. Incidence and mortality of gastric cancer in China. World J Gastroenterol. 2006; 12:17-20.

[2] Fock KM, Ang TL. Epidemiology of Helicobacter pylori infection and gastric cancer in Asia. J Gastroenterol Hepatol. 2010; 25:479-86.

[3] International Agency for Research on Cancer. GLOBOCAN [Internet]. 2012 [cited 2017 Jul 05]. Available from: http://globocan. iarc.fr/

[4] Bertuccio P, Chatenoud L, Levi F, Praud D, Ferlay J, Negri E, et al Recent patterns in gastric cancer: a global overview. Int J Cancer. 2009; 125:666-73.

[5] Hussein NR. Helicobacter pylori and gastric cancer in the Middle East: A new enigma? World J Gastroenterol. 2010; 16:3226-34.

[6] Malekzadeh R, Derakhshan MH, Malekzadeh Z. Gastric cancer in Iran: epidemiology and risk factors. Arch Iran Med. 2009; 12:576-83.

[7] Haidari M, Nikbakht MR, Pasdar Y, Najaf F. Trend analysis of gastric cancer incidence in Iran and its six geographical areas during 2000-2005. Asian Pac J Cancer Prev. 2012; 13:3335-41.

[8] Matsuhisa T, Aftab H. Observation of gastric mucosa in Bangladesh, the country with the lowest incidence of gastric cancer, and Japan, the country with the highest incidence. Helicobacter. 2012; 17:396-401.

[9] Howlader N, Ries LA, Stinchcomb DG, Edwards BK. The impact of underreported Veterans Affairs data on national cancer statistics: analysis using population-based SEER registries. J Natl Cancer Inst. 2009; 101:533-6.

[10] Zeraati H, Amiri Z. Estimating postoperative survival of gastric cancer patients and factors affecting it in Iran: based on a TNM-7 Staging System. Acta Med Iran. 2016; 54:114-8.

[11] Adham D, Abbasgholizadeh N, Abazari M. Prognostic factors for survival in patients with gastric cancer using a random survival forest. Asian Pac J Cancer Prev. 2017; 18:129-34.

[12] Dikshit RP, Mathur G, Mhatre S, Yeole BB. Epidemiological review of gastric cancer in India. Indian J Med Paediatr Oncol. 2011; 32:3-11. 
[13] Dickman PW, Coviello E. Estimating and modeling relative survival. Stata J. 2015; 15:186-215.

[14] Dickman PW, Adami HO. Interpreting trends in cancer patient survival. J Intern Med. 2006; 260:103-17.

[15] Lambert PC, Dickman PW, Osterlund P, Andersson T, Sankila R, Glimelius B. Temporal trends in the proportion cured for cancer of the colon and rectum: a population-based study using data from the Finnish Cancer Registry. Int J Cancer. 2007; 121:2052-9.

[16] Lambert PC, Thompson JR, Weston CL, Dickman PW. Estimating and modeling the cure fraction in population-based cancer survival analysis. Biostatistics. 2007; 8:576-94.

[17] Eloranta S, Lambert PC, Andersson TM, Bjorkholm M, Dickman PW. The application of cure models in the presence of competing risks: a tool for improved risk communication in population-based cancer patient survival. Epidemiology. 2014; 25:742-8.

[18] Andersson TM, Dickman PW, Eloranta S, Lambert PC. Estimating and modelling cure in population-based cancer studies within the framework of flexible parametric survival models. BMC Med Res Methodol. 2011; 11:96. doi: 10.1186/1471-2288-11-96

[19] Cronin KA, Feuer EJ. Cumulative cause-specific mortality for cancer patients in the presence of other causes: a crude analogue of relative survival. Stat Med. 2000; 19:1729-40.

[20] Lambert PC, Dickman PW, Nelson CP, Royston P. Estimating the crude probability of death due to cancer and other causes using relative survival models. Stat Med. 2010; 29:885-95. 